\title{
Maximum Output Amplitude of Linear Systems for certain Input Constraints
}

\author{
Wolfgang Reinelt \\ Department of Electrical Engineering \\ Linköping University, S-581 83 Linköping, Sweden \\ WWW: http://www.control.isy.liu.se/ ºlle/ \\ Email: wolle@isy.liu.se
}

June 1999

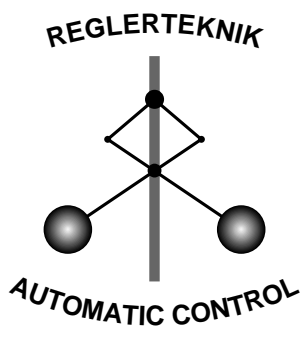

LINKÖPING

Report no.: LiTH-ISY-R-2165

Technical reports from the Automatic Control group in Linköping are available by anonymous ftp at the address ftp.control.isy.liu.se. This report is contained in the portable document format file 2165.pdf. 


\title{
Maximum Output Amplitude of Linear Systems for certain Input Constraints
}

\author{
Wolfgang Reinelt
}

June 1999

\begin{abstract}
We determine the maximum output amplitude of a system, when its input fulfils certain constraints. In particular, the amplitude and the rate of change (i.e. the first derivative) have to be bounded. We show properties of the worst case input and present an algorithm that allows to construct this input and calculates the maximum amplitude of the output. This problem has applications within the non-conservative design of controllers for plants with bounded inputs. Nevertheless, it is interesting as a system theoretic task itself and therefore stated seperately.
\end{abstract}

Keywords: Linear System Theory, Maximum Output Amplitude, Hard Constraints.

\section{Introduction and Motivation}

Linear systems with bounded inputs produce bounded outputs, stability provided. Given the maximum amplitude of the input and calculating the maximum amplitude of the output is the problem of determining the 1-norm of the system. A more challenging task is to calculate the maximum output amplitude for a system with inputs that fulfil certain additional constraints.

For motivation we look at process engineering for example, and in particular at the liquidlevel in a tank, interpreted as a time signal. Not only the liquid-level in a tank is bounded (by the tank's height), additionally the liquid cannot change its level arbitrarily fast. So this signal is bounded in amplitude, as well as its first derivative due to the time. Regarding the liquid-level as a reference signal for a control system with control signal let's say the opening angle of some valve (feeding the tank) it is quite reasonable to design a controller that takes care for the possible reference signals for the liquid level as well as for the amplitude bound for the control signal. The bound for the control signal is, loosly speaking, the range between fully open and fully closed.

Interpreting this in system-theoretic terms, the transfer function from reference signal to control signal is a system with an input that fulfils certain constraints (bounds for amplitude and first derivative) and a bounded output signal. Task of the controller design is now to design the controller so that the maximum amplitude of the control signal is not higher than "fully open" in order to prevent wind-up and stability problems. Therefore it is obviously helpful to calculate the maximum output amplitude for a given system with prescribed input constraints on amplitude and first derivative.

At a first glance it might be sufficient to neglecet the constraint on the reference signal's speed and solve the problem for arbitrarily fast changing reference signals. As these 
non-smooth reference signals are impossible in most applications it becomes clear, that the resulting controller will be too conservative. During run-time, the amplitude of the control signal will be much smaller than the allowed one; this effect will obviously reduce the control systems's speed and performance.

However, the solution of the technical problem has many applications in the design of controllers that guarantee bounded control signals [1]. In particular, optimal control problems can be solved $[4,5]$, as well as robust control problems [6, 7]. The term optimal refers to the fact, that the constraint $|\dot{r}(t)| \leq \dot{R}$ allows a minimization of the error signal $|e(t)|=|r(t)-y(t)|$. An extension for the discrete time case is possible [3, 2, 8]. These control applications will be presented seperately.

This work is organized as follows: section 2 defines the problem, which will then be solved in section 3. Section 4 sketches shortly the numerical realisation. The ideas are extended to the multivariable case in section 5, suited for multivariable control systems. Section 6 illustrates the theory with an example. Conclusions are drawn in section 7 , related works as well as the direction of future research are also given. Problem statement and solution are due to $[1,4]$, a numerical implementation was outlined in [5] and the extension to the multivariable case was given in $[6,7]$.

\section{Problem Statement}

We examine a linear and time invariant stable system, which is represented by its transfer function $G(s)$ resp. its impulse response $g(t)$. We postpone the extension to the multivariable case to section 5 and concentrate on the SISO case. The input is denoted by $r$, the output by $x$. The following constraints hold for the continuous and piecewise ${ }^{1}$ differentiable input signal $r$ :

$$
\begin{aligned}
& |r(t)| \leq R \\
& |\dot{r}(t)| \leq \dot{R}
\end{aligned}
$$

for $t>0$, where $R, \dot{R}>0$ are given constant values and

$$
r(t)=0, \quad t \leq 0 .
$$

We call those reference signals, which fulfil eqns.(1-3) $(R, \dot{R})$-admissible, or short $r \in \mathcal{A}(R, \dot{R})$. We are looking for the maximum amplitude $X_{m}(t)$ of the output $x$ (up to a certain time) with $(R, \dot{R})$-admissible inputs, i.e.

$$
X_{m}(t):=\sup _{r \in \mathcal{A}(R, \dot{R})} \sup _{0<\tau \leq t}|x(\tau)|=\sup _{r \in \mathcal{A}(R, \dot{R})} \sup _{0<\tau \leq t}|g(\tau) \star r(\tau)| .
$$

where " $\star$ " is the convolution: $g(t) \star r(t)=\int_{0}^{t} g(\tau) r(t-\tau) d \tau$. Quite clear from linear system theory is, that for systems with the only input constraint (1), the maximum output amplitude is given by $\max _{t \geq 0}|x(t)|=R \int_{0}^{\infty}|g(\tau)| d \tau$, produced by the bang-bang input:

$$
r(t-\tau)=R \cdot \operatorname{sign}(g(\tau)) .
$$

Thus the problem is trivial unless the additional constraint (2) is imposed.

\footnotetext{
${ }^{1}$ a countable number of non-differentiable time stamps $t$ is allowed.
} 


\section{Properties of the Worst Case Input}

We now turn to the construction of the maximum output amplitude as stated in eqn.(4) according to $[4]$. Basic idea is first to show some properties of the input signal $r$, which produces the output with the maximum output amplitude. In the following, we call this input signal the worst case input. This strategy is motivated by the existence of a worst case input in the simple case in eqn.(5). We start with the calculation of the output by convolution for a certain time stamp $t$ :

$$
x(t)=\int_{0}^{t} g(\tau) r(t-\tau) d \tau=: \int_{0}^{t} g(\tau) r_{t}(\tau) d \tau
$$

and abbreviate the "time inverted" input signal by $r_{t}(\tau):=r(t-\tau)$ with the following consequences for the constraints (1-3):

$$
\begin{aligned}
\left|r_{t}(\tau)\right| & \leq R, \quad \tau<t \\
\left|\dot{r}_{t}(\tau)\right| & \leq \dot{R}, \quad \tau<t \\
r_{t}(\tau) & =0, \quad \tau \geq t
\end{aligned}
$$

3.1 Lemma The function $X_{m}(t)$ is monotone increasing (i.e. not decreasing) in time $t$. Therefore, the maximum amplitude as defined in eqn.(4) appears for sure for $t \rightarrow \infty$, so $X_{m}=$ $\lim _{t \rightarrow \infty} X_{m}(t)$ is the maximum output amplitude.

Proof. Let $t_{0}>0$ and $r_{t_{0}} \in \mathcal{A}(R, \dot{R})$ the input ${ }^{2}$ that produces the maximum amplitude $X_{m}\left(t_{0}\right)$. For $t_{1}>t_{0}$ define $r_{t_{1}}(\tau)=r_{t_{0}}(\tau), 0 \leq \tau \leq t_{0}$ resp. $r_{t_{1}}(\tau)=0, t_{0}<\tau \leq t_{1}$. Clearly $r_{t_{1}} \in \mathcal{A}(R, \dot{R})$ and from eqn.(6) follows sup $\operatorname{su}_{0<\tau \leq t_{1}}|x(\tau)|=\sup _{0<\tau \leq t_{0}}|x(\tau)|$ and thus $X_{m}\left(t_{1}\right) \geq X_{m}\left(t_{0}\right)$.

3.2 Definition Suppose there exists an input $r_{\infty, o}=: r_{o}$ with maximum output amplitude $X_{m}$. Then $-r_{o}$ produced obviously the maximum output amplitude $X_{m}$, too. For one of them, say $r_{\mathrm{o}}$, holds

$$
X_{m}=\int_{0}^{\infty} g(\tau) r_{o}(\tau) d \tau \geq 0
$$

i.e., in eqn.(4) the absolute value is superfluous. In the following, we construct this worst case input ${ }^{3}$ that produces this maximum output amplitude according to eqn.(10).

3.3 Algorithm (Construction of an auxiliary input) Let $r \in \mathcal{A}(\mathrm{R}, \dot{\mathrm{R}})$ be an arbitrary admissible input. We construct an auxiliary input $r_{H}$ for $r$ uniquely by the steps given below (figure 1 illustrates the construction). The set of all possible auxiliary inputs (i.e. all signals with the same properties) is denoted by $\mathcal{A}_{\mathrm{H}}(\mathrm{R}, \dot{\mathrm{R}})$.

1. Let $t_{i}, i=1, \ldots, N$ the zeros of $g$. Define $r_{H}\left(t_{i}\right)=r\left(t_{i}\right)$.

2. If $g(t)>0$ in $\left(t_{i}, t_{i+1}\right)$, let $\dot{r}_{H}(t)=+\dot{R}$ in the neighbourhood of $t_{i}$ and $\dot{r}_{H}(t)=-\dot{R}$ in the neighbourhood of $t_{i+1}$. In the case that this definition leads to the non-unique situation that the two 'slopes' intersect in some $t_{*} \in\left(t_{i}, t_{i+1}\right)$, let $\dot{r}_{H}(t)=+\dot{R}$ in $\left[t_{i}, t_{*}\right]$ resp. $\dot{r}_{H}(t)=-\dot{R}$ in $\left[t_{*}, t_{i+1}\right]$. Define finally $r_{H}=\min \left\{r_{H},+R\right\}$.

\footnotetext{
${ }^{2}$ uniqueness is not necessary for this argumentation.

${ }^{3}$ with index "o" for optimal.
} 


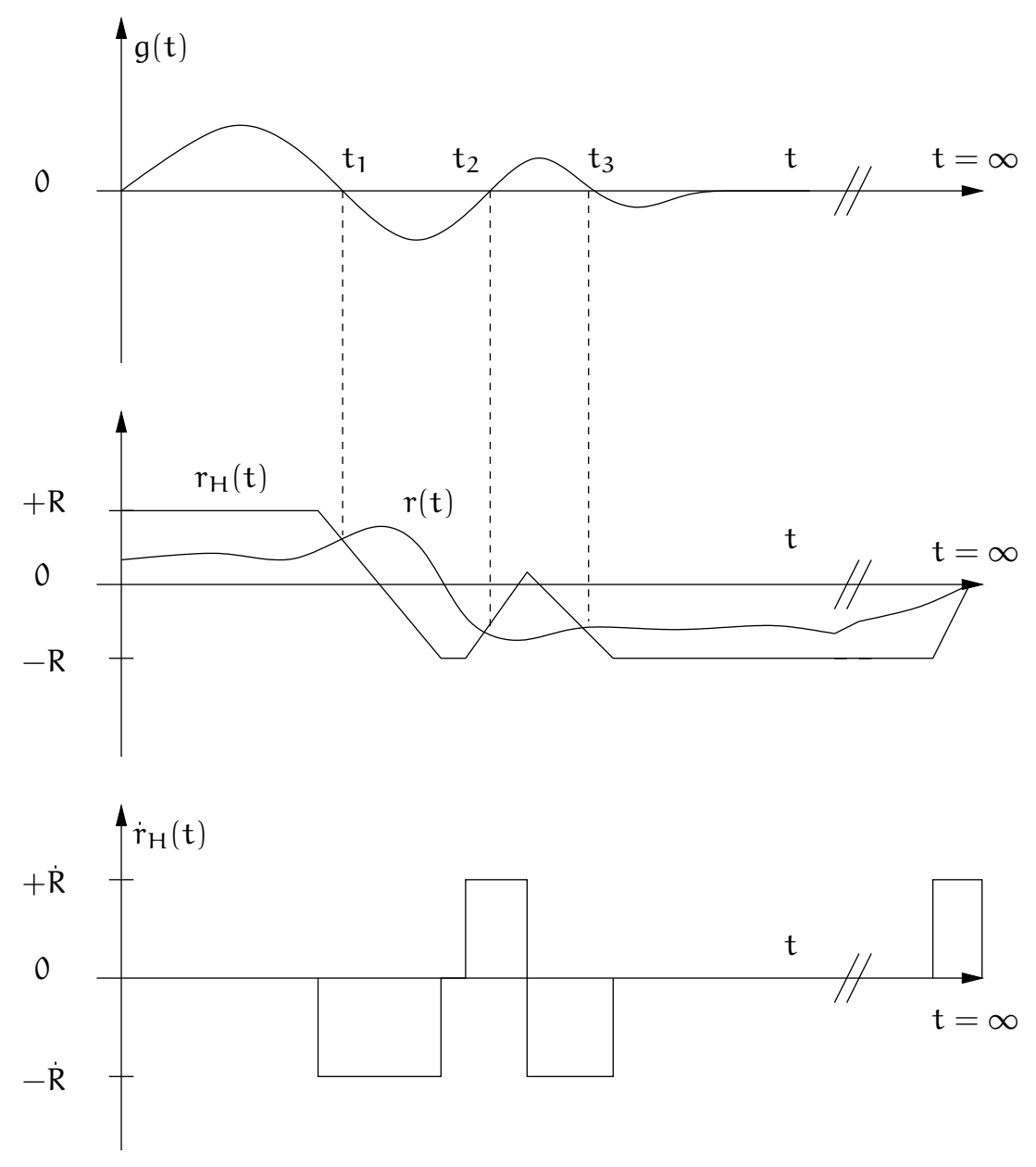

Figure 1: Construction of auxiliary input $r_{H}$ for given input $r$.

3. If $g(t)<0$ in $\left(t_{i}, t_{i+1}\right)$, do as in step 2. but with changed signs for $\dot{r}_{H}$ and resulting obvious modifications.

4. Finally, choose $\left|\dot{r}_{H}(t)\right|=\dot{R}$ for large times $t$ in order to ensure $\lim _{t \rightarrow \infty} r_{H}(t)=0$, arising from constraint (9).

3.4 Corollary the following properties of the auxiliary input are clear by construction:

1. $\mathcal{A}_{\mathrm{H}}(\mathrm{R}, \dot{\mathrm{R}}) \subset \mathcal{A}(\mathrm{R}, \dot{\mathrm{R}})$, i.e. an auxiliary input is also admissible.

2. Two different inputs $r_{1}, r_{2} \in \mathcal{A}(R, \dot{R})$ with $r_{1}\left(t_{i}\right)=r_{2}\left(t_{i}\right)$ have the same auxiliary input.

3. $r_{H}(t) \geq r(t)$ for $g(t) \geq 0$ and $r_{H}(t) \leq r(t)$ for $g(t) \leq 0$.

4. Fix an admissible input $r$ and suppose an arbitrary admissible signal $r^{*} \neq r_{H}$ with property 3., then $\int_{0}^{\infty} g(\tau) r_{H}(\tau) d \tau>\int_{0}^{\infty} g(\tau) r^{*}(\tau) d \tau$.

5. The maximum width of the pulses of $\dot{r}_{H}$ in Algorithm 3.3 is given by $T=2 \frac{R}{\dot{R}}$.

3.5 Theorem The worst case input is auxiliary input: $r_{\mathrm{o}} \in \mathcal{A}_{\mathrm{H}}(\mathrm{R}, \dot{\mathrm{R}})$. 
Proof. For all $r \in \mathcal{A}(R, \dot{R})$ the following holds by construction of $r_{H}$, see Corollary 3.4 (3.):

$$
\int_{0}^{\infty} g(\tau) r(\tau) d \tau \leq \int_{0}^{\infty} g(\tau) r_{H}(\tau) d \tau
$$

and "=" holds only for $r \equiv r_{H}$ (except the case $g \equiv 0$ ). Assume $r_{o} \in \mathcal{A}(R, \dot{R}) \backslash \mathcal{A}_{H}(R, \dot{R})$, then the construction of an auxiliary input $r_{\mathrm{oH}}$ is possible (because $r_{\mathrm{o}}$ is admissible input). Applying eqn.(11) to $r=r_{o}$ :

$$
X_{m}=\int_{0}^{\infty} g(\tau) r_{o}(\tau) d \tau<\int_{0}^{\infty} g(\tau) r_{o H}(\tau) d \tau
$$

which contradicts the definition of $X_{m}$ as the maximum output amplitude. Consequently $\mathrm{r}_{\mathrm{o}} \in \mathcal{A}_{\mathrm{H}}(\mathrm{R}, \dot{\mathrm{R}})$.

Until now, we did not construct a unique worst case input that leads to the maximum output amplitude, but we showed some necessary properties which are listed in the following

3.6 Lemma The following necessary properties of the worst case input $r_{o}$ hold:

1. The (derivative of the) worst case input has a pulse-shape: $\dot{r}_{o}(t) \in\{ \pm \dot{R}, 0\}$, where $\dot{r}_{o}(t)=$ 0 implies $\left|r_{\mathrm{o}}(\mathrm{t})\right|=\mathrm{R}$.

2. The width of the single pulses is constrained by $T=2 \frac{R}{\bar{R}}$.

3. Two adjacent pulses have different signs.

4. $\lim _{t \rightarrow \infty} r_{o}(t)=0$ and $\left|\lim _{t \rightarrow \infty} \dot{r}_{o}(t)\right|=\dot{R}$.

The previous Lemma states mostly properties of $\dot{\mathrm{r}}_{\mathrm{o}}$. Partial integration of eqn.(10) together with Lemma 3.6 (4.) gives an expression for the maximum output amplitude in these terms, where $s$ is the step response of the system (i.e. $\dot{s}=g$ ):

$$
X_{m}=-\int_{0}^{\infty} s(\tau) \dot{r}_{o}(\tau) d \tau
$$

Despite the minus in eqn.(13), $X_{m} \geq 0$ holds by Definition 3.2. It appears only due to the partial integration.

Looking onto eqn.(13) and knowing the shape of the worst case input as stated in Lemma 3.6, the solution is quite clear: in order to make the integral maximal, put some pulses (of maximum width, see Corollary 3.4 (4.)) in the near of extrema of the step reponse: positive ones in the near of the minima and negative ones in the near of the maxima. In the following, we will prove this. In order not to overload the discussion with technical details, we make the following 


\subsection{Temporary Assumption}

1. Let the impulse response $g$ have only a finite number $N$ of zeros, i.e. the step response only a finite number of extrema.

2. Let the first extremum of $s$ to be a (local) maximum.

Under these assumptions, the maximum amplitude $X_{m}$ is given by:

$$
X_{m}=\dot{R} \sum_{i=1}^{N}(-1)^{i+1+k} \int_{t_{i}^{\prime}}^{t_{i}^{\prime \prime}} s(t) d t+R(-1)^{N+k} \lim _{t \rightarrow \infty} s(t) .
$$

The last part of the sum exists because the system is stable. The pairs $\left(t_{i}^{\prime}, t_{i}^{\prime \prime}\right)$ refer to the unknown positions of the pulses. Additionally, the sign of the pulses is still unknown, therefore we added $k \in\{0,1\}$ in eqn.(14). Obviously the problem is solved, when we know the exact location of the pulses and their sign. We make one more

3.8 Temporary Assumption Let the extrema of the step response have a distance $>2 \mathrm{~T}$, which ensures that all pulses have maximum width $T$, i.e. $t_{i}^{\prime \prime}=t_{i}^{\prime}+T$.

3.9 Theorem Necessary condition for a maximum $s\left(t_{i}^{\prime}\right)=s\left(t_{i}^{\prime}+T\right)$, sufficient condition is $k=0$ in eqn.(14).

Proof. Necessary for a maximum is $\frac{\partial X_{m}}{\partial t_{i}^{\prime}}=0$ for all $i$, which leads immediateley to $s\left(t_{i}^{\prime}\right)=s\left(t_{i}^{\prime}+\right.$ $T)$. Sufficient is that the Hessian is negative definite, which leads directly to $\dot{R}(-1)^{i+k}\left(g\left(t_{i}^{\prime}+\right.\right.$ $\left.\mathrm{T})-\mathrm{g}\left(\mathrm{t}_{\mathfrak{i}}^{\prime}\right)\right)<0$ thus $\mathrm{k}=0$, because of assuming a first local maximum of $s$ in Assumption 3.7.

Applying Theorem 3.9 to eqn.(14), we get the maximum output amplitude by

$$
X_{m}=\dot{R} \sum_{i=1}^{N}(-1)^{i+1} \int_{t_{i}^{\prime}}^{t_{i}^{\prime}+T} s(t) d t+R(-1)^{N} \lim _{t \rightarrow \infty} s(t) .
$$

where we place the pulses $\left(t_{i}^{\prime}, t_{i}^{\prime}+T\right)$, so that $s\left(t_{i}^{\prime}\right)=s\left(t_{i}^{\prime}+T\right)$ holds with a negative sign under the maxima of $s$ and with a positive sign under the minima of $s$.

3.10 Remark on temporary assumptions 3.7 and 3.8:

1. The stability of the system ensures $g(t) \rightarrow 0$ for large $t$, i.e. $s(t)=$ const. In the case, that $\mathrm{g}$ has a infinite number of zeros the maximum output amplitude is given by eqn.(15) with an arbitrary precision when using arbitrary many zeros (i.e. $\mathrm{N} \rightarrow \infty$ ) For an exact proof, we refer to [4, Appendix A2.1].

2. Suppose a first local minimum for $s$. In this case, the sufficient condition would lead to $k=1$, i.e. to a change of signs for all pulses. We can simply treat these different cases by taking the absolute value of the sum in eqn.(15).

3. The "well-distinctness" of the extrema, assumed in Assumption 3.8 ensures that pulses with width $T$ posed around the extrema of $s$ will not intersect each other. Technically, this assumption simplifies the proof of Theorem 3.9. In general, we can prove that $\int_{t_{i}^{\prime}}^{t_{i}^{\prime \prime}} g(t) d t=0$ is necessary and sufficient for a maximum. The proof is of technical nature and brings no deeper insight. We refer to [4, Appendix A2.2] for the details. 
In practice, this means that an odd number of extrema, located in an interval of length $\mathrm{T}$, is viewed as one maximum resp. minimum. For the entire discussion of all cases, we refer again to [4, Appendix A2.2].

3.11 Remark For $\dot{R} \rightarrow \infty$ (i.e. no restriction on the input's speed), the worst case input converges to the bang-bang input, as given in eqn. (5).

\section{Numerical Algorithm}

Theorem 3.9 and Remark 3.10 give us necessary and sufficient conditions to construct the worst case input. The maximum output amplitude can be calculated with help of the worst case input and eqn.(15). We restrict ourselves on a not very formal description of the algorithm that constructs the worst case input numerically. Numerical details and the handling of special cases are outlined in [5].

1. Determine extrema of the step response.

2. Put initial pulses with correct and alternating signs under each extremum of $s$.

3. Make them wider, ensuring $s\left(t_{i}^{\prime}\right)=s\left(t_{i}^{\prime \prime}\right)$, as long as they do not intersect.

4. In the case of intersections, handle "not well distinct" pulses separately.

5. Calculate the maximum output amplitude from eqn.(15).

The problem can also be viewed as a nonlinear optimisation problem in the pulse positions: determine $t_{i}^{\prime}, t_{i}^{\prime \prime}$, so that rhs of eqn.(15) becomes maximal. Indeed, a solution by nonlinear optimisation is possible, having a good initial value given by step (2.) of the procedure above; this solution is outlined in [6].

\section{Multivariable Case}

We extend the previous result to the case of multivariable systems, i.e. $r$ and $x$ are now vector signals [6]. What we have in mind with this extension is the treatment of multivariable control systems with constraint control signals, i.e. we regard $x=u$ and $r$ is the (external) reference signal of the control system. Therefore, it is useful to restrict the input $r$ componentwise:

$$
\begin{aligned}
|r(t)| & \preceq R, t>0 \\
|\dot{r}(t)| & \preceq \dot{R}, t>0 \\
r(t) & =0, t \leq 0 .
\end{aligned}
$$

in analogy to eqns.(1-3). Read $\preceq$ as a componentwise $\leq$ and evaluate $|\cdot|$ therefore componentwisely. Consequently, we call the set of all signals $r$ fulfilling these constraints $(R, \dot{R})$ admissible, with $R, \dot{R}$ are now being vectors with positive entries. Furthermore, we define the maximum output amplitude componentwise by

$$
X_{m}:=\left(X_{1, m}, \ldots, X_{n, m}\right)^{\top}
$$


where the $X_{i, m}$ are defined as in Lemma 3.1.

Let us first look onto a system with one output $x$ and $k$ inputs $r=\left(r_{1}, \ldots, r_{k}\right)^{\top} \in \mathcal{A}(R, \dot{R})$. Then $x(s)$ is given by

$$
x(s)=G_{1}(s) \cdot r_{1}(s)+\cdots+G_{k}(s) \cdot r_{k}(s)
$$

We abbreviate $\tilde{x}_{i}(s)=G_{i}(s) \cdot r_{i}(s)$. Now we are looking for the maximum output amplitude $X_{m}$. Using eqn. (20), the maximum output amplitude is given by

$$
X_{m}=\sum_{i=1}^{k} \tilde{X}_{i, m} .
$$

It follows directly, that $X_{m}$ is achieved for a certain vector $r=\left(r_{1}, \ldots, r_{k}\right)^{\top} \in \mathcal{A}(R, \dot{R})$.

In the multivariable case with $n$ outputs, we simply apply the first step: according to the definition, the components $X_{i, m}$ of $X_{m}$ can be calculated as in equation (21).

\section{Illustrative Example}

We examine the system represented by the transfer function

$$
\mathrm{G}(\mathrm{s})=\frac{\mathrm{s}^{2}+0.4}{\mathrm{~s}^{2}+1.4 \mathrm{~s}+1.0}
$$

with input constraints $R=1.0$ resp. $\dot{R}=0.8$. According to Lemma 3.6 (2.), the maximum pulse width is $T=2.5$. Numerical solution by construction of the worst case input yields

$$
X_{m}=0.76 \text {. }
$$

Fig. 2 shows the construction of the worst case input with pulses of $r_{0}$ located at $[0,1.20]$, $[1.20,2.39],[5.24,7.74]$ and at of width $\mathrm{T} / 2$ at infinity (in reversed time) with signs " - + +" following max-min-max sequence of the step response. For simulation of this worst case input we need to reverse this reversed time, therefore we choose the "infinite" time to $t_{\infty}=120$. With this transformation, we receive the depicted worst case output with a maximum amplitude of $x\left(t_{m}\right)=0.73$ for $t_{m}=108.80$ as a good approximation for the maximum amplitude as calculated in eqn.(22).

\section{Conclusions and Related Works}

We showed conditions of the worst case input with bounded amplitude and speed, that produces that maximum output amplitude for a given (stable) multivariable system. A numerical algorithm was sketched to construct this worst case input and to calculate the maximum output amplitude. This is, as we believe, an important step within the non-conservative controller design for systems with hard bounds on the control signal. Minimisation of the error signal in a control system is possible with these assumptions on the reference signal. Moreover it enables us to check the maximum amplitude an arbitrary signal within the control system after controller design. These control applications will be presented in sequel. 


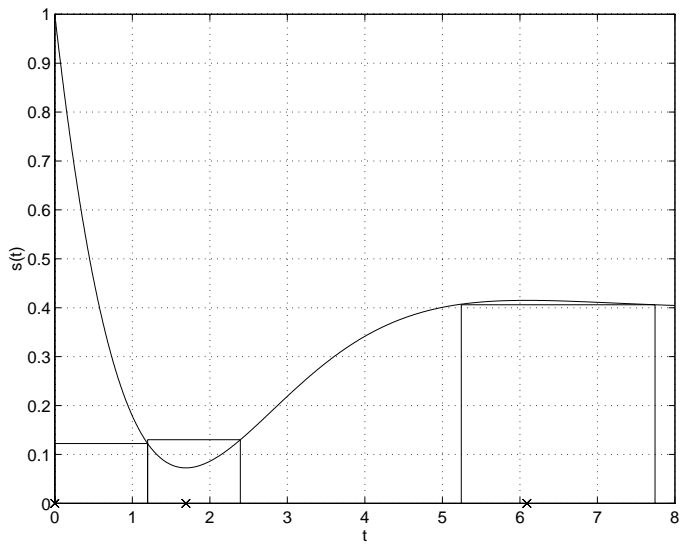

(a) Step response $s$ with calculated position of the pulses for the worst case input.

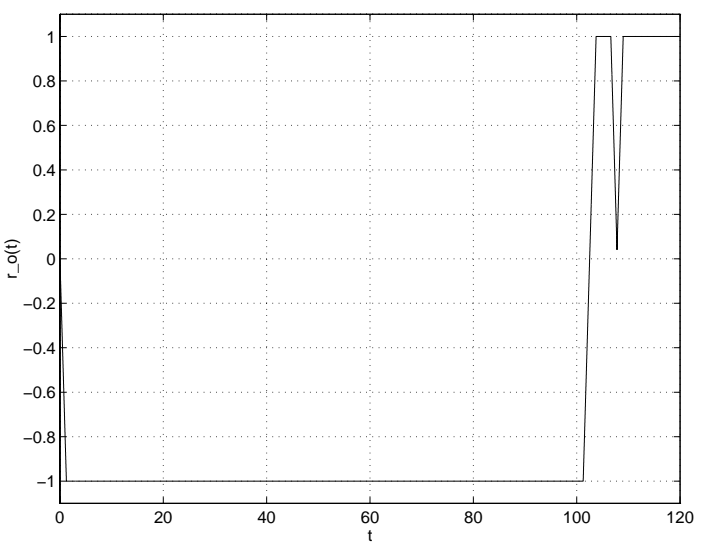

(b) Worst case input.

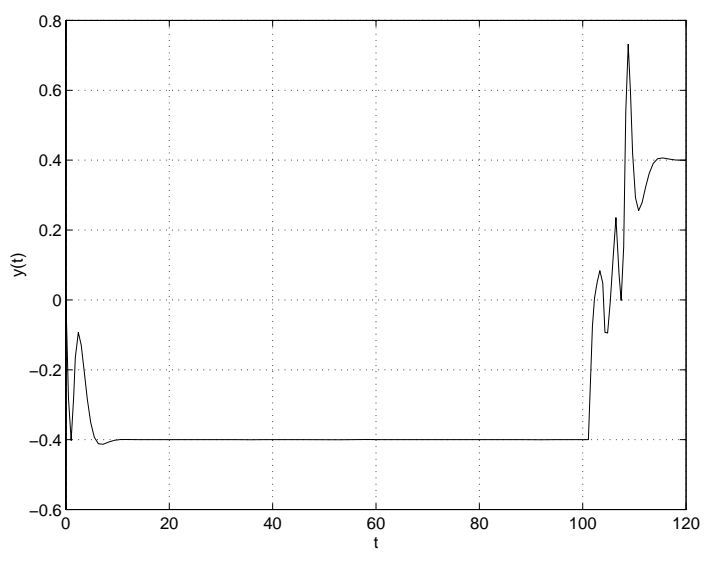

(c) Worst case output.

Figure 2: Illustrative example: construction of the worst case input and output. 


\section{References}

[1] N. Dourdoumas. Prinzipien zum Entwurf linearer Regelkreise mit Beschränkungen eine Einführung. Automatisierungstechnik, 35(8):301-309, Aug. 1987.

[2] P. Müller. Entwurf optimaler Abtastregelkreise bei beschränkten Systemgrössen. Master's thesis, Dept of EE, University of Paderborn, 33095 Paderborn, Germany, 1994.

[3] X. Peng. Rechnerunterstützte Synthese von Abtastregelkreisen mit Beschränkungen. PhD thesis, Dept of EE, University of Paderborn, 33095 Paderborn, Germany, 1992.

[4] R. W. Reichel. Synthese von Regelsystemen mit Beschränkungen bei stochastischen Eingangsgrössen. PhD thesis, Dept of EE, University of Paderborn, 33095 Paderborn, Germany, 1984.

[5] W. Reinelt. Entwurf optimaler Regler bei amplitudenbeschränkten Systemgrössen. Master's thesis, Dept of Math, Univ of Paderborn, 33095 Paderborn, Germany, Dec. 1993.

[6] W. Reinelt. Robust Control of Systems subject to Hard Constraints. PhD thesis, Dept of EE, Univ of Paderborn, 33095 Paderborn, Germany, Apr. 1998.

[7] W. Reinelt. $\mathcal{H}_{\infty}$ loop shaping for systems with hard bounds. In Proc. of the Int Symp on Quantitative Feedback Theory and Robust Frequency Domain Methods, Durban, South Africa, pages 89-103, Aug. 1999.

[8] I. Zacharias. Robuste Regelung von Abtastsystemen unter Berücksichtigung von Stellgrössenbeschränkungen. Master's thesis, Dept of Math, University of Paderborn, 33095 Paderborn, Germany, Feb. 1999. 